\title{
Automatic Detection of Plant Disease and Insect Attack using EFFTA Algorithm
}

\author{
Kapilya Gangadharan ${ }^{1}$ \\ Research Scholar, Dept. of Computer Science and \\ Engineering, SSE, Saveetha Institute of Medical and \\ Technical Sciences Chennai, India
}

\author{
G. Rosline Nesa Kumari ${ }^{2}$ \\ Professor, Dept. of Computer Science and Engineering \\ Shadan women's college of Engineering and Technology, \\ Telangana, India
}

\author{
D. Dhanasekaran ${ }^{3}$ \\ Professor, Dept. of Computer Science and Engineering \\ SSE, Saveetha Institute of Medical and Technical Sciences \\ Chennai, India \\ K. Malathi ${ }^{4}$ \\ Associate Professor, Dept. of Computer Science and \\ Engineering, SSE, Saveetha Institute of Medical and \\ Technical Sciences Chennai, India
}

\begin{abstract}
The diagnosis of plant disease by computer vision using digital image processing methodology is a key for timely intervention and treatment of healthy agricultural procedure and to increase the yield by natural means. Timely addressal of these ailments can be the difference between the prevention and perishing of an ecosystem. To make the system more efficient and feasible we have proposed an algorithm called Enhanced Fusion Fractal Texture Analysis (EFFTA). The proposed method consists of Feature Fusion technique which combines SIFT- Scale Invariant Feature Transform and DWT- Discrete Wavelet Transform based SFTA- Segment Based Fractal Texture Analysis. Image as a whole can be detected by shape, texture and color. SIFT is used to detect the texture feature, it extracts the set of descriptors that is very useful in local texture recognition and it captures accurate key points for detecting the diseased area. Further extraction of texture is considered and that can be performed by WSFTA method. It adopts intra- class analysis and inter- class analysis. Extracted features trained using Back Propagation Neural Network. It improves and expands the success rate and accuracy of extraction also it provides higher precision and efficiency when compared to the other traditional methods.
\end{abstract}

Keywords-Texture analysis; features; computer vision; interclass; intra-class

\section{INTRODUCTION}

Digital images are typically represented in the form of texture, shape and color features. It indicates the attributes of the image. Dimensions of the raw data or information can be reduced by extracting the features. It is a process that is very much required in Machine Learning and pattern recognition. Where the required texture attributes are extracted from the specified dataset. The characteristic represents the original data property like texture, shape or color based upon the requirement. Texture is based on the feature and shape is based on the template Feature extraction mainly deals with reducing the large number of data into subset that describes the required information. The process makes the classification simpler and accurate. Machine learning methodology is mainly dependent on the appropriate and efficient feature extraction. Extraction begins with an underlying arrangement of already estimated information and determined values or features planned as instructive and non-repetitive, it accelerates the successive learning and speculation steps, also it is interpreted by human as well [16]. And it is identified with dimensionality reduction. SIFT process converts the image into smaller dimensions with the required information. It is mainly used for object matching criteria. Key point extraction through SIFT is more accurate than the other traditional methods, improvement shows $11.12 \%$ more using SIFT [17]. SFTA is a used for image decomposition which utilizes OTSU thresholding in a conventional method. To make the decomposition much easier we follow Discrete Wavelet Transform and it is passed to STFA algorithm for further decomposition and it creates a hybrid method called WSFTA. When shift is combined with WSFTA it produces a best and accurate result and it is relatively easier for the classifier to recognize the diseased portion and the healthy portion of the plants. Our work mainly concentrates on proposing the enhanced texture feature fusion which combines SIFT and WSFTA, once after fusing the algorithms it is very important to perform selection process. Selection is done using PCA method to avoid dense feature vector creation that elaborates the vector length causing high computational cost [19]. The selection process results in the accurate and required features. The selected features are then trained and tested using Back Propagation Neural Network Classifier.

\section{RELATED WORK}

The texture parameters are calculated using gray level synchronizing matrix spatial variants and to define the diseased area in the plant leaves [1]. Bark classification on texture and fractal dimension is proposed. It combines texture and the structural features to improve the accuracy [2]. Another method is proposed using the objective values, it is calculated by Kurtosis, variance, Skewness and Entropy, where homogeneity and contrast is calculated for estimating the diseased area [3]. GLCM (Gray level Covariance Matrix) is used to extract the texture features where 12 types of features are extracted which include Entropy, Skewness, Kurtosis, Smoothness, Variance, etc. [4]. A method called 
HGPASO and it is combined with OCGR method is used to reduce the steps which is carried out to calculate the threshold by Multilevel OSTU method [5].

Image is processed using traditional Image processing methods and SIFT algorithm is passed through the preprocessed image to extract the color feature, where SIFT texture feature is described using Johnson SB distribution. Average precision is calculated using cross validation of 10fold [6]. Analysis of key point extraction is done using SIFT and SURF extraction methods, it is proved that SIFT process is more accurate than SURF. The improvement is shown $11.12 \%$ more than SURF. It is classified using neural network classifier [7]. Shrinking Edge detection method is proposed and applied on recursive support vector mechanism to classify the image and to estimate the performance of the system [8]. Color histogram detects the diseased area of the image where other features are combined to generate the classification [9]. To classify and detect the diseased parts in the leaf images various methods like SIFT, HOG, Pyramid histogram word, Dense SIFT are compared [10]. SFTA extraction is composed based on the wavelet transformation, where image is decomposed into multiple sub bands using wavelet transformation and Texture feature is extracted using SFTA algorithm [11].

\section{PROPOSED SYSTEM}

Feature extraction is an important tool in the Image processing field. Multiple features can be extracted from the digital image, the features referred as color, shape and texture. the plants can suffer from biotic plant diseases it could be due to pest attack or any kind of infection from fungus, bacteria or Virus. When there is a presence of disease or infection on any region of the plant say, leaf or bud or stem the diseased region will appear in different degree. Based on the difference in the characteristic of the diseased region we can estimate the change in color, shape or texture feature, also it can recognize and show the variation in each type of infection or pest attack. Before performing feature extraction method basic image processing techniques like Image acquisition, standardization, preprocessing.

Segmentation [15] should be performed so that extracting features would be much simpler and accurate. Fig. 1 describes the basic workflow of the proposed system. We have used multiple feature extraction methods to make the system more feasible. Performed Feature using SIFT and WSTFA algorithm. Feature fusion is a special technique that we have adapted in our proposed system it combines the extracted features of multiple methods using Enhanced Fusion Fractal Texture Analysis (EFFTA) algorithm.
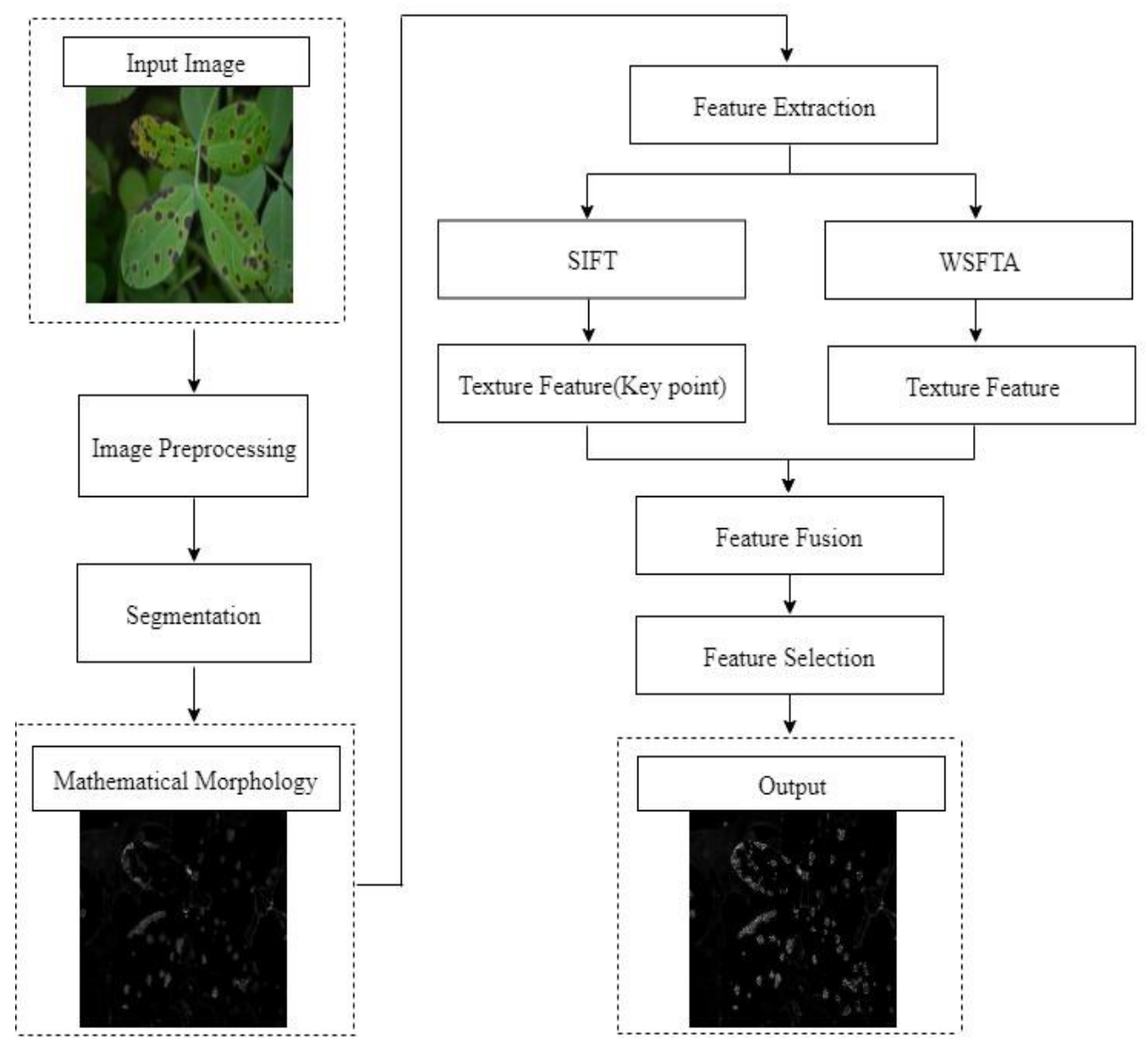

Fig. 1. Proposed System Architecture. 


\section{A. Proposed Algorithm}

Step 1: Pick the image (Horizontal Gradient) extracted after Morphological Operation (Gray scaled Image)

\section{Step 2: Apply SIFT Process:}

a) Create an internal depiction of input image to check the scale variance through scale space.

b) Laplacian of Gaussian (LoG) approximation is used to find the key points. Since it is very expensive, we use DoG.

c) Calculate Difference of Gaussian (DoG), difference in Maxima and minima to estimate the key points. feature.

d) Locate the extreme points and assign the SIFT

e) Avoid or eliminate the Edges and low contrast i.e. poorly employed key points and appropriate key points are calculated.

f) Responsible key points must be assigned with orientation. Any further calculations will be done based on the orientation.

g) More depictions are generated which will be easy to identify the features.

h) Add the key points to a common list

Step 3: Mapping Discrete Wavelet Transform

a) Decompose input image into different frequency.

b) 2D-DWT is performed by applying 1D-DWT.

c) 1D-DWT is applied on the row of the Image and it is decomposed along the column.

d) If the result is 4 decomposition then the sub bands generated are LL, LH, HL and HH.

$e$ ) High pass filters produce detailed information on each level.

f) Scale factor is established by using Low pass filter.

g) Half band filter extracts signal spanning at each decomposition level. It multiplies the frequency intense into two and the unreliability in the frequency is reduced. (SFTA)

Step 4: Segmentation Based Fractal Texture Analysis

a) Low frequency sub band images are examined, which decomposes the image into binary image.

b) To decompose LL Image extracted from DWT, Two Threshold binary decomposition (TTBD) is utilized.

c) Using Fractal proportion from the examined region edges for every extracted binary image are calculated.

d) Finally, average of gray level and dimensions of region i.e., the boundaries(pixels) are calculated.

e) Add the extracted Wavelet based SFTA key points to a common list.

Step 5: Feature fusion is done for the features extracted by SIFT and WSFTA

a) Through the response value sort the common list. b) Select the appropriate feature (diseased region) using Serial Based method.

Step 6: Perform Step 1 through Step 5 until all the features are extracted.

\section{B. SIFT Key Point Extraction}

Scale Invariant Feature Transform is an efficient algorithm that detects the local features of an image by extracting the Key points. The extracted Key points specifies the exact location and its descriptors. SIFT key points are extracted first from the set of reference images and it is saved in the database. The regions are perceived in the input image by independently comparing each component from the input image from the database. The detecting point of interest or component is the key point. A scale space is produced to represent scale variance in the input image.

Gaussian filter is applied on the Image under different scenario (scaling factor) and the difference obtained by the image which is blurred after applying Gaussian filter is taken. The Minimum and Maximum value from the difference is computed with multiple scaling factor which is also known as Difference of Gaussian (DoG).

$D(x, y, \sigma)=L\left(x, y, \sigma_{i}\right)-L\left(x, y, \sigma_{j}\right)$

$L(x, y, \sigma)=G(x, y, \sigma) * I(x, y)$

Where, $D(x, y, \sigma)$ is the DoG Image, $L(x, y, \sigma)$ is the convolution, $\sigma_{i}, \sigma_{j}$ is the Gaussian Blur $I(x, y)$ is the scaling factor of an image. The difference between $\sigma_{i}$ and $\sigma_{j}$ computed for different scales under multiple blurred condition on each pixel. The key points are differentiated with the threshold ranges 0.03 , if the contrast is smaller than the threshold it is eliminated as low contrast values. DoG eliminates the edge with more accuracy, if the contrast is higher than the threshold the edge key points are rejected. So, it terminates the low contrast key point and boundary key point and it maintains only the strongest key points. Then each key point is allocated to more than one position using gradient factor extracted from the morphological operations when the positions are established. Then the magnitude and direction of each pixels are calculated with its neighboring pixel values. From which a histogram is generated, and the highest peak of the histogram is assigned to the key point, where more depictions are generated so as to make the system more feasible to identify the required features.

\section{Feature Extraction using WSFTA Algorithm}

Wavelet based SFTA feature extraction technique is proposed [12] to detect the infectious region from the input image. To effectively extract the texture feature we combine Discrete Wavelet Transform and SFTA as shown in the Fig. 2. The acquired feature vector will be the input for the classification algorithm to examine if the input image is identified with disease or not. Combining Wavelet Transform and SFTA algorithm it extracts 30 features from the low frequency sub bands. 


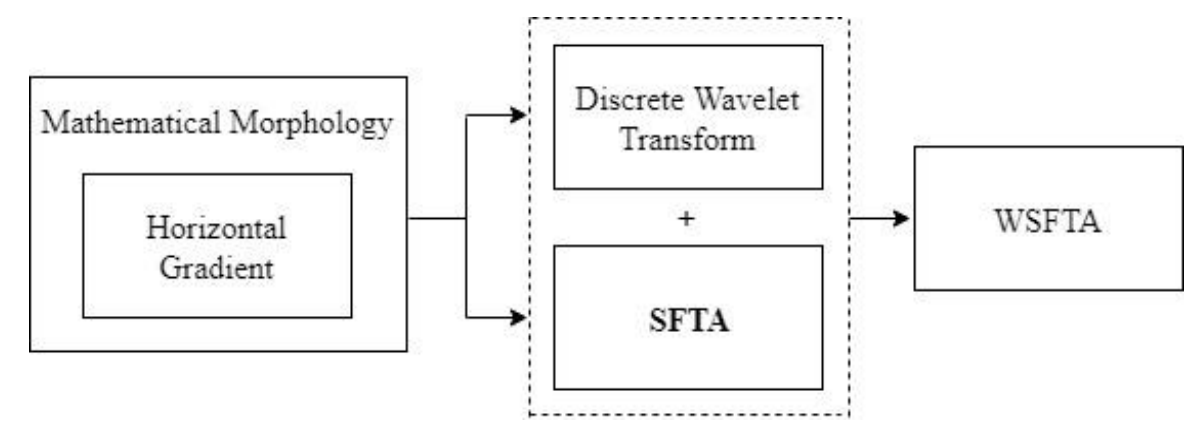

Fig. 2. Architecture of WSFTA.

a) Discrete Wavelet Transform: Feature extraction is one of the predominant aspects of classification. In this system, the extracted gradient through morphological method is sub divided into various frequency bands by applying Wavelet transform. DWT is a transformation tool that has ample of applications like feature extraction, compression, normalization, etc. It captures the frequency as well as the location information like texture, shape, etc. 2D-DWT decomposes the sample into four major sub bands, which is performed by 1D-DWT on the row of the input sample and then it is decomposed towards the column.

The result of 1D-DWT decomposition produces four types of sub band, they are Low Low (LL), Low High (LH), High Low (HL) and High High (HH). If wavelet functions are separable i.e., $f(x, y)=f_{1}(x) f_{2}(y)$, the scaling factor is low level frequency then the component of the previous scaling function is 2 dimension, which implies there is one 2D scaling factor and There are different wavelet functions $\psi^{H}(x, y)$, $\psi^{V}(x, y)$ and $\psi^{D}(x, y)$ for 2D scaling and filter can be applied to the wavelet function [13].

$\phi(x, y)=\phi(x) \phi(y)$

$\psi^{H}(x, y)=\psi(x) \phi(y)$

$\psi^{V}(x, y)=\phi(x) \psi(y)$

$\psi^{D}(x, y)=\psi(x) \psi(y)$

2D filters $H_{L L}, H_{L H}, H_{H L}$ and $H_{H H}$ are obtained from eq (1) to (4) and that corresponds to $\phi, \psi^{H}, \psi^{V}, \psi^{D}$ respectively. Although 2D approach involves more operation than 1D filtering this method is very efficient in implementing the feature extraction of low-level images.

$f(x, y)=\frac{1}{\sqrt{M N}} \sum_{m}\left(\sum_{n}\left(W_{\phi}\left(j_{0}, m, n\right) \phi_{j_{0}, m, n}(x, y)\right)\right.$
$+\frac{1}{\sqrt{M N}} \sum_{i=H, V, D}\left(\sum_{j=0}^{\infty}\left(\sum_{m}\left(\sum_{n}\left(W_{\psi}^{i}(j, m, n) \phi_{j, m, n}^{i}(x, y)\right)\right)\right)\right.$

Where

$W_{\phi}\left(j_{0}, m, n\right)=\frac{1}{\sqrt{M N}} \sum_{x=0}^{M-1}\left(\sum_{y=0}^{N-1}\left(f(x, y) \phi_{j_{0}, m, n}(x, y)\right)\right)$

$W_{\psi}^{i}(j, m, n)=\frac{1}{\sqrt{M N}} \sum_{x=0}^{M-1}\left(\sum_{y=0}^{N-1}\left(f(x, y) \phi_{j, m, n}^{i}(x, y)\right)\right)$

and $i=\{H, V, D\}$ b) Segmentation Based Fractal Texture Analysis (SFTA): SFTA Algorithm is mainly used to decompose the gray scaled image into multiple binary images and the output would be segmented regions which are used to define the texture patterns. So, that it will be easy for us to identify the diseased region and a normal region. The algorithm is split into two main specifications: 1. Two threshold binary decomposition in binary images. 2. SFTA calculates the fractal dimension from the boundaries of the regions. Also, pixels should be counted from all the extracted regions.

TTB Decomposition method: It takes gray scaled image $I(x, y)$ and split it into multiple binary images, where $I(x, y)=\left\{1,2,3,4, \ldots n_{t}\right\}$. Initially, Compute threshold value $T$, Threshold is determined by computing the equally spaced gray values in the image, Where the values range between 1 to $n_{t}$. It is done using multilevel OTSU method. The Multilevel OTSU method computes the threshold by minimizing the image intra-class variance and it is applied to each region that is split until the exact threshold is extracted.

$I_{b}(x, y)=\left\{\begin{array}{c}1 \text { if } I(x, y) \geq T \\ 0, \text { Otherwise }\end{array}\right.$

Where $I_{b}(x, y)$ is the binary image, $T$ is the threshold and set the threshold obtained by equally spaced gray level value, which is called as One-Threshold Segmentation. Then the next step is to splits the gray scale image $I(x, y)$ into binary images. For which the extracted pair of thresholds Multiple $T$ is selected and apply as Two-threshold segmentation, which computes the inter-class variance.

$I_{b}(x, y)=\left\{\begin{array}{c}1 \text { if } T_{A}<I(x, y) \leq T_{B} \\ 0, \text { otherwise }\end{array}\right.$

Where $T_{A}$ and $T_{B}$ represents lower and upper threshold, after the threshold computation SFTA vector is constructed over it.

SFTA Extraction Algorithm: SFTA vector is constructed once after the threshold is computed using Two-Threshold segmentation. Where the boundaries of the fractal dimension and mean gray level is computed. The geometric phenomenon is engaged to explain the complexity and severity of the boundaries and the segmented structure. The boundary regions are $I_{b}(x, y)$ and the border image as $\delta(x, y)$.

$\delta(x, y)=\left\{\begin{array}{c}1 \text { if } I(x, y) \in N_{30}(x, y) \\ I_{b}(x, y)=0 \\ 0, \text { otherwise }\end{array}\right.$ 
Where $N_{30}$ is the pixel that has 30 connect to $(x, y)$. If the pixel is in the position $(x, y)$, then $\delta(x, y)$ picks the value 1 from the binary image $I_{b}(x, y)$, which is set to 1 with at least one neighboring pixel as 0 , else $\delta(x, y)=0$.

\section{Algorithm:}

Step1: Threshold $T \leftarrow$ Multilevel OTSU $\left(I, n_{t}\right)$ Step2: Threshold set 1 from binary Image $A \leftarrow$ $\left\{\left\{t_{i}, t_{i+1}\right\}: t_{i}, t_{i+1} \in T, i \in[1,2,3, \ldots[T]-1]\right\}$ Step3: Threshold set 2 from binary Image $B \leftarrow$ $\left\{\left\{t_{i}, n_{l}\right\}: t_{i} \in T, i \in[1,2,3, \ldots[T]]\right\}$

Step 4: $i \leftarrow 0$

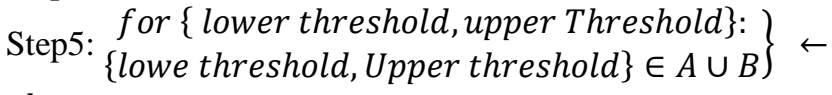
do

Step 6: Binary Image $I_{b}(x, y) \leftarrow$

Two threshold segmentation

(I, lower threshold $T_{A}$, Upper threshold $T_{B}$

Step 7:

Border Image $\delta(x, y) \leftarrow$

Find borders of $I_{b}$ (Binary Image)

Step 8:

SFTA Feature Vector $[i] \leftarrow$

Count box of $\delta(x, y)$ (Border Image)

Step 9:

SFT A Feature Vector $[j+1] \leftarrow$

Mean gray level $\left(I, I_{b}\right.$ (Binary Image $\left.)\right)$

Step 10:

SFT A Feature Vector $[j+2] \leftarrow$

Pixel count of $I_{b}$ (Binary Image)

Step 11: $i \leftarrow i+3$

Step 12: end for

Step 13: Return SFTA Feature Vector

\section{Feature Fusion Methodology}

It is an advanced method that is carried out in research to combine multiple features in one vector [12]. It upgrades the performance and precision level when compared with the individual analysis. Robust and the efficient features are combined by calculating the similarity between the extracted features on the same region. The fusion of multiple features has the objects on their own representation. It could be texture, shape color etc. Combining various features extracted by multiple methods provides better result and accuracy.

In our proposed system as show in Fig. 3 we combined the features extracted by SIFT algorithm is placed in a common list and likewise pixel count extracted by the WSFTA is placed in a common list. Fusion is done using Serial-Based Method. $f_{n}=\left\{n / n \in R^{S I F T}\right\}$ is the SIFT feature vector with dimension 1 X 2800 and $f_{m}=\left\{\mathrm{m} / \mathrm{m} \in R^{\text {WSFTA }}\right\}$ is the WSFTA vector with dimension $1 \mathrm{X} 30 . m, n$ are the feature vector. which is calculate using the sample data $\phi$ that perform training and testing. Let $\Delta=(\phi / \phi) \in R S$ and fusion can be done as $\alpha \mathrm{f}=n m$.
We get the vector values from the common list when the features of SIFT and WSFTA is placed. $f_{n}$ is the vector for SIFT and $f_{m}$ is the vector for WSFTA. So, we calculate $f_{v}=\left\{f_{n}+f_{m}\right\}$ where the dimensions of the extracted vectors are known. Feature selection is carried out by adding the minimum distance and Pearson co-efficient. The selection method produces perfect and flawless feature from the combined vector, and it minimizes the elapsed time during execution. The selection method involves Euclidian distance (ED) calculation. Where threshold $\mathrm{T}$ is computed from the combined feature vector, extracted through the minimum distance feature $D\left(f_{v}\right)$. Mean of Minimum distance is used to calculate the skewness which requires the median value from the given set of vectors and the variance of Minimum distance.

$$
E=-\sum_{v}\left(f_{v} \log _{2}\left(f_{v}\right)\right)
$$

Entropy is calculated by obtaining Euclidian Distance, Minimum Distance, Skewness and Variance where PCA is applied on the extracted features for obtaining optimized features. Select the highest score from the calculated entropy to recognize the best feature of diseases part of the plant which is on observation. The Training algorithm considered in this proposed method is Back Propagation Neural network. Classifier which recognizes the infection by training and testing process.

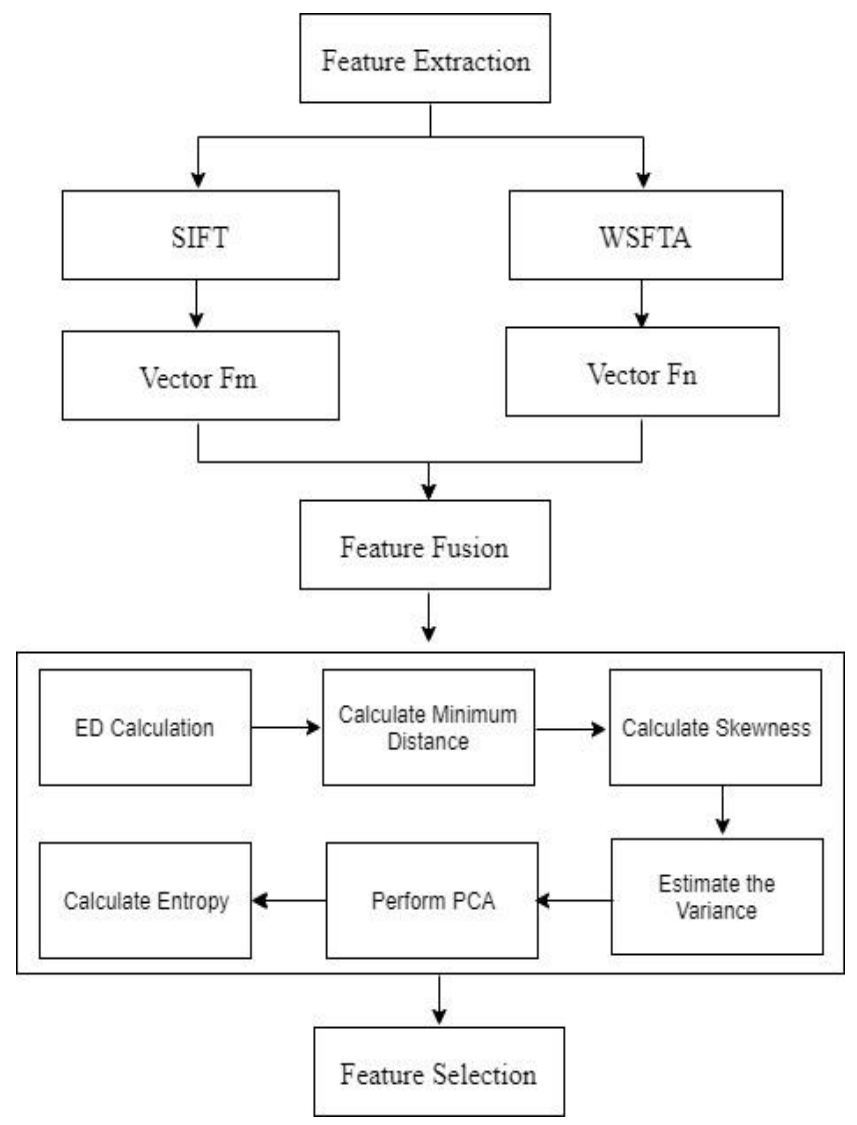

Fig. 3. Feature Fusion Method. 


\section{E. Classification Process}

The selected features are trained and tested using Back Propagation Neural Network Technique. Where $80 \%$ of the image from the dataset is trained and $20 \%$ of the image from the dataset are tested using BPNN. Back propagation calculates the missed errors by performing back loops. It is based on the principle square of Euclidean distance and the calculated entropy. The internal memory is used for processing the output sequence and it showcases the dynamic temporal behavior of the system. Grading the learning system is the most important factor. For better and accurate result an optimum number should be considered for grading. Momentum is multiplied for the number of backward iterations so as to find the discrepancies in the hidden layer.

\section{Algorithm:}

Step 1: Allot the required input and Output

Step 2: Consider the weight from the range -1 and 1 .

Repeat Step 2 for all the available pattern in the training object.

Step 3: Initial value is predicted by performing Forward propagation.

Step 4: The discrepancies in the hidden layers are identified through backpropagation.

Step 5: Calculate local and global error.

Step 6: Repeat the steps until (maximum number of iterations $<$ specified) and (Error function $>$ specified).

Step 7: Increase the momentum from -0.5 to 0.5 and conduct multiple training sessions.

\section{EXPERIMENTAL RESULTS AND DISCUSSION}

The proposed method is tested and analyzed on various plant diseases and pest attacks using the dataset captured from internet $[14,18]$. The experimental results include the performance of individual algorithm and the fusion and selection method. we have tested our algorithm using MATLAB to code and executed on Windows 10, 64-bit Operating System with $8 \mathrm{~GB}$ Ram, $2.70 \mathrm{GHz}$ Intel Core i5 7th Gen Processor.

\section{A. Performance Comparison on Scaling and other Factors}

At first, we have evaluated the performance of the Fusion texture by comparing it with individual algorithm by changing the scale ranges from 0.25 to 2.0. From Fig. 4, it is observed that the proposed method Feature Fusion which is the combination of SIFT and WSFTA algorithm obtained correct match better than the other existing individual algorithms like SIFT, SFTA, BRISK, WSFTA. We have picked the normal and changed the scaling factor to test the correct match. We have tested on multiple images and taken average matching factor from total number of 300 Images with multiple diseases. The enhancement on the precision is better and accurate using proposed algorithm. Which has the robust factor for detecting the feature by extracting the maximum number of Key points.

The same way matching criteria is calculated using other factors like Rotation, Affine transform, Illumination, Gaussian Blur effect. These tests are carried out to check if we can get exact match on the proposed system, because we have fused more than one algorithm, while combining more than one texture feature method it is very important to notice if it produces good matching criteria. We adapted similarity matching factor using Serial Based method. Rotation is another major factor that is used in feature detection. We have compared the set of images with multiple degree of rotation. And it is observed that proposed method excelled in various rotation criteria starting from 30 to 60 degree as shown in Fig. 5.

Also, when the test was performed by applying affine transform, Gaussian blur and Illumination the proposed method produced better match when compared to the existing methods which is depicted in Fig. 5. When the algorithms are combined it produces better result than the individual processing.

\section{B. Key Point Extraction}

Extracting key point is one of the critical and important aspect in feature detection or extraction. In our proposed system before feature fusion, we have collected the extracted features through Key points using SIFT method. We have compared SIFT, SURF and BRISK on the number of key points extracted on a specific threshold. The observation shows that the key points obtained by applying, provides the better ways to detect and classify the diseased portion. Average key points extracted are shown in Fig. 6. Higher number of key points extracted produces better result. When we compared the three methods it is proven that SIFT produces higher key points than the other two methods.

\section{Feature Analysis}

The feature extraction of various images and diseases are calculated with reference to multiple texture features. We have extracted the features for multiple diseases like Cercospora leaf spot on Peanut leaves, Bacterial Blight disease on Paddy leaves and few pest attacks like, Boll Weevil on Cotton buds, European Corn Borer on Cotton leaves and Fall army Worm on the peanut leaves. We have calculated the scores of the extracted features separately by using the fusion method. Where few features are examined like ED, Variance, Skewness, Kurtosis and Entropy. After the feature extraction we have spotted the diseased area by selecting the features. Calculate entropy value for each feature and select the highest score among the list and the highest score is used for recognition. Table I shows the average calculated scores. And the highest Entropy values for individual diseases. We have used more than 300 Images for the calculation.

\section{Implementation Result}

The input images are collected from the datasets available in the internet [14]. In the future we are planning to use the image captured in drone with high resolution camera. We have performed morphological operations on the segmented image and selected the horizontal gradient. In this paper we have used the extracted Horizontal Gradient Image as the input image and we have performed SIFT operation to extract the key point and decomposition is done by Wavelet transform and SFTA Algorithm and extracted the texture features. And both the algorithms are combined using Fusion feature method and based on the highest Entropy value we have selected the diseased region as shown in Fig. 7 to Fig. 11. 


\section{E. Comparitive Evaluation}

The proposed feature extraction technique is compared with the traditional state of art methods with slight variation in the dataset. Overall accuracy is considered for comparison since the identified disease using the other traditional methods are different. The proposed system is trained using BPNN and the other tradition methods are trained using SVM and BPNN. From the analysis it is determined that the proposed system produces better result with the accuracy of $97.69 \%$ as shown in Table II.

The performance has been evaluation of the proposed system is based on the valuation metrics. It can be classified as

True Positive (TP): Abnormal case appropriately evaluated as Abnormal.
False Negative (FN): Abnormal case imperfectly classified as normal.

False Positive (FP): Normal case imperfectly identified as abnormal.

True Negative (TN): Normal case appropriately calculated as Normal.

Sensitivity: TP/TP+FN

Specificity: TP/TP+FP

Accuracy: $(\mathrm{TN}+\mathrm{FP}) /(\mathrm{TN}+\mathrm{TP}+\mathrm{FN}+\mathrm{FP})$

F-Score: $(2 *$ Sensitivity*Specificity)/(sensitivity+specificity)

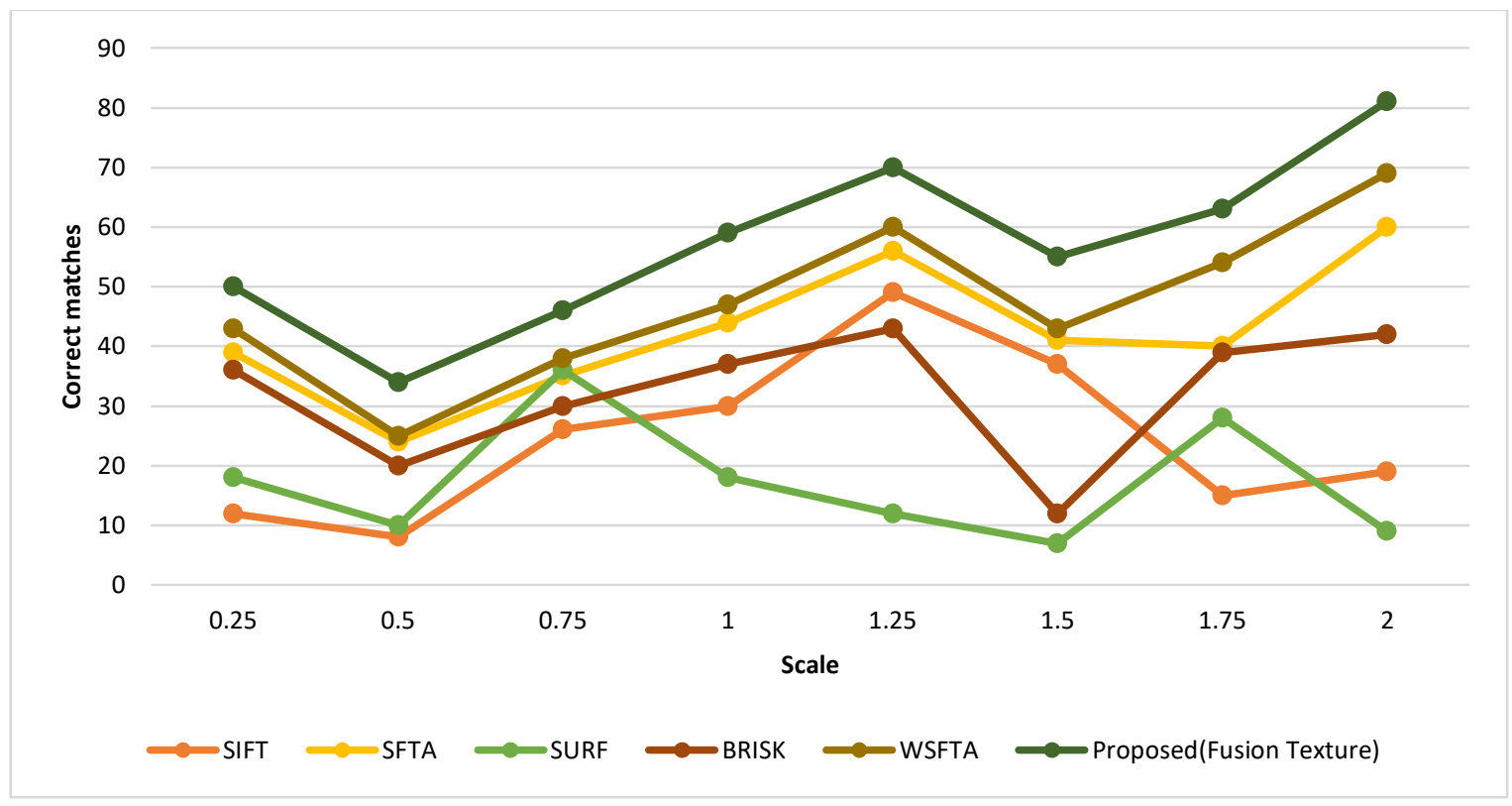

Fig. 4. Performance Comparison using Multiple Scaling Fctor.

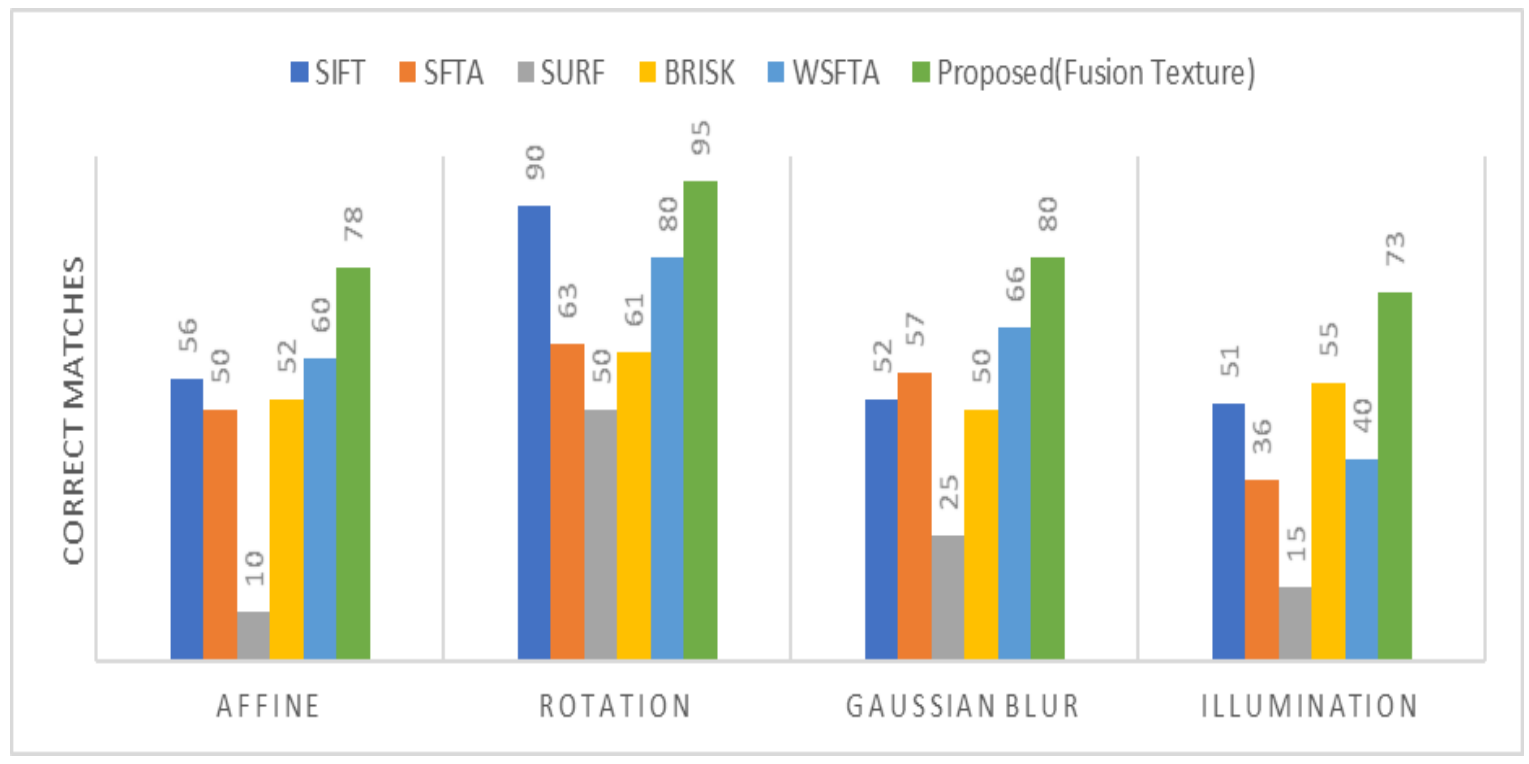

Fig. 5. Performance Evaluation using Multiple Factors. 


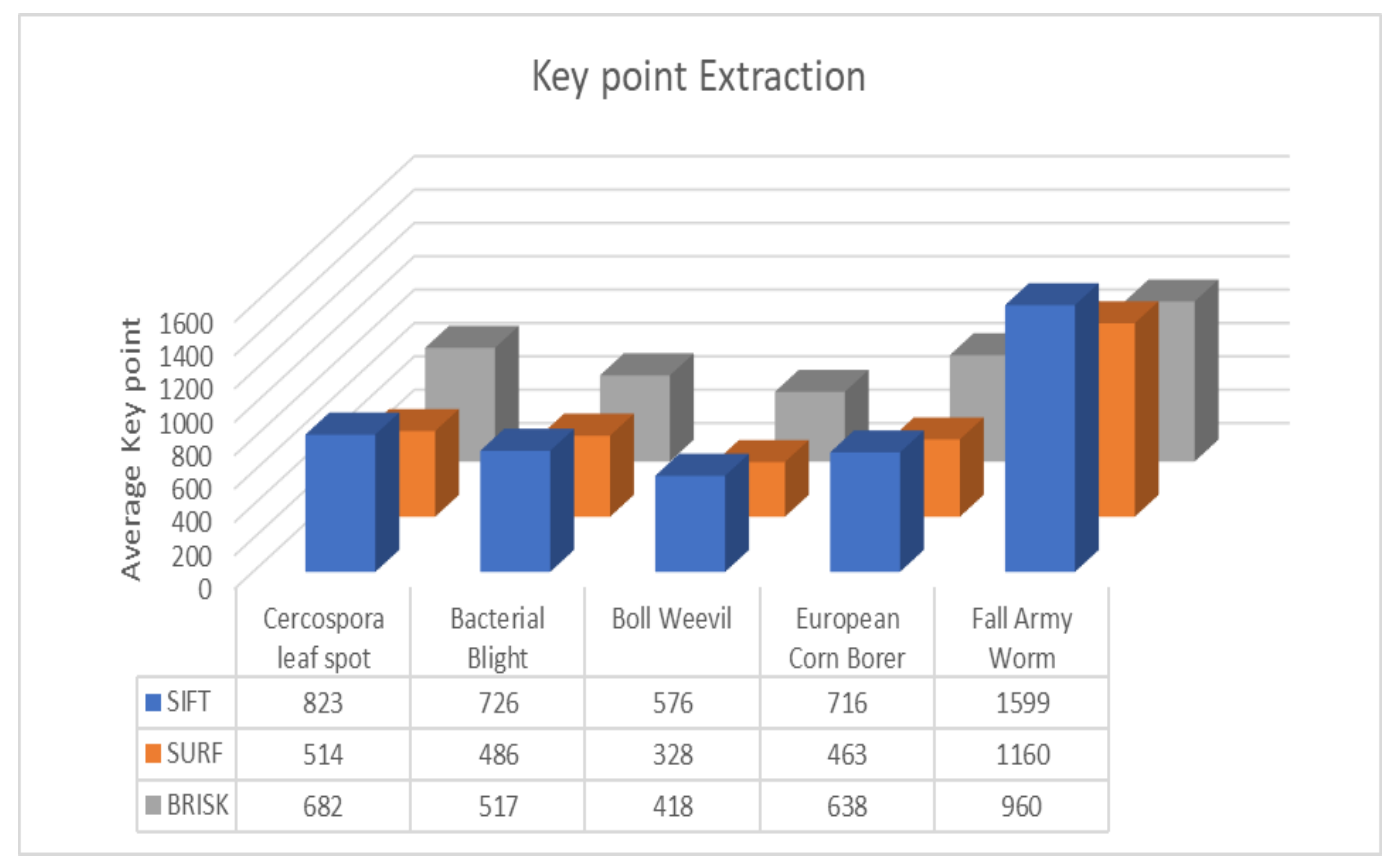

Fig. 6. Comparison of Key Point Extraction.

TABLE. I. EXTRACTED FEATURES

\begin{tabular}{|l|l|l|l|l|l|}
\hline \multirow{2}{*}{ Features } & Peanut leaves & Paddy Leaves & Cotton Buds & Cotton leaves & Peanut leaves \\
\cline { 2 - 6 } & Cercospora leaf spot & Bacterial Blight & Boll Weevil & European Corn Borer & Fall Army Worm \\
\hline Euclidian distance & 48.3456 & 23.8312 & 11.5674 & 17.6571 & 33.6172 \\
\hline Variance & 45.7434 & 27.8767 & 15.5633 & 19.4415 & 35.9787 \\
\hline Skewness & 1.5001 & 2.5143 & 4.2657 & 2.9349 & 4.1015 \\
\hline Kurtosis & 4.6343 & 8.4565 & 22.7562 & 10.2745 & 15.3851 \\
\hline Entropy & 4.1004 & 2.5647 & 1.2109 & 1.9111 & 3.154 \\
\hline
\end{tabular}

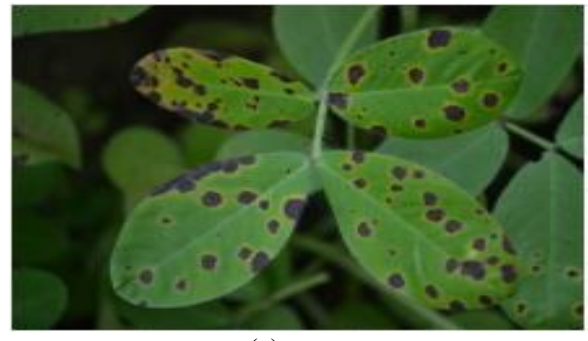

(a)

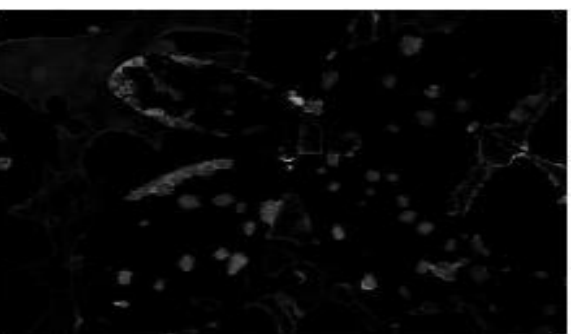

(b)

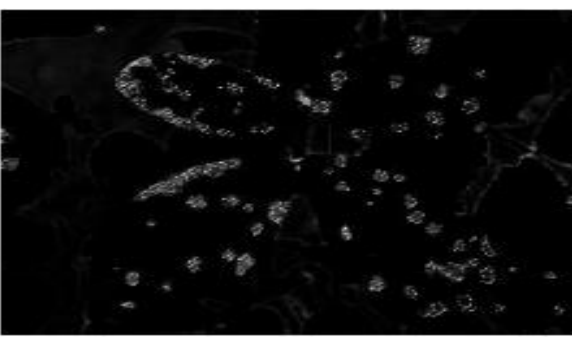

(c)

Fig. 7. Feature Extraction of Cercospora Leaf Spot on Peanut Leaves (a) Input Image (b) Horizontal Gradient Extracted by Mathematical Morphology (c) Extracted Features using Proposed Method.

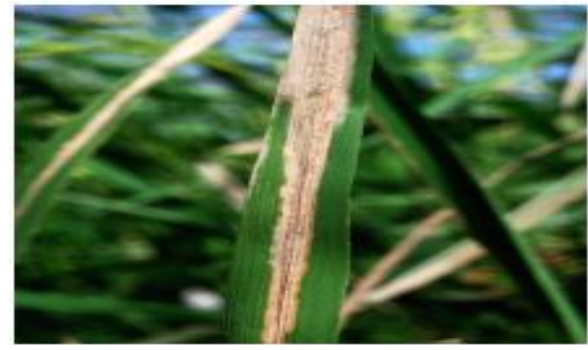

(a)

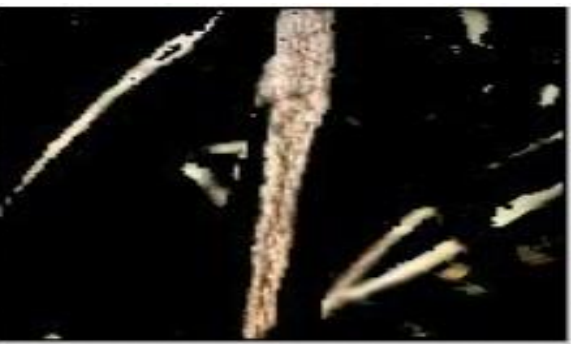

(b)

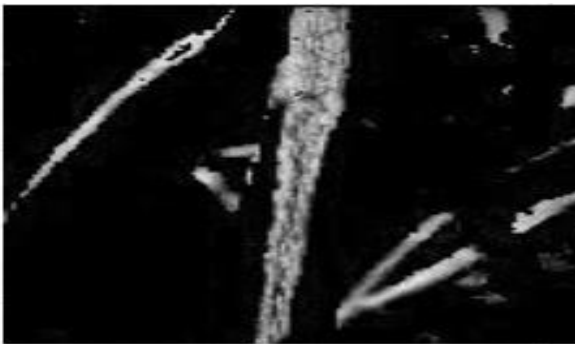

(c)

Fig. 8. Feature Extraction of Bacterial Blight Disease on Paddy Leaves (a) Input Image (b) Horizontal Gradient Extracted by Mathematical Morphology (c) Extracted Features using Proposed Method. 


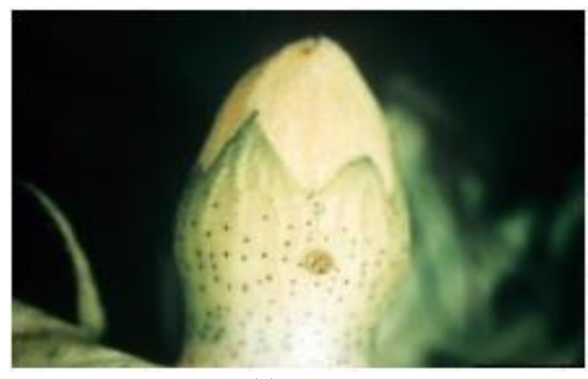

(a)

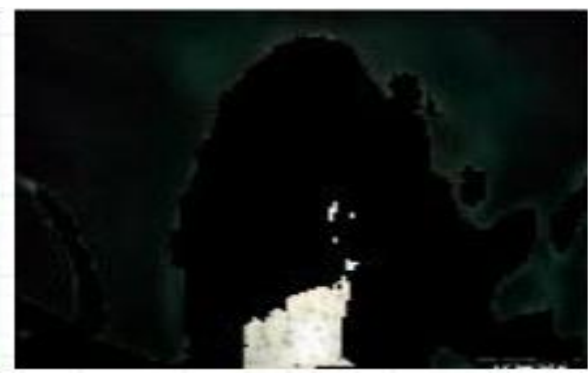

(b)

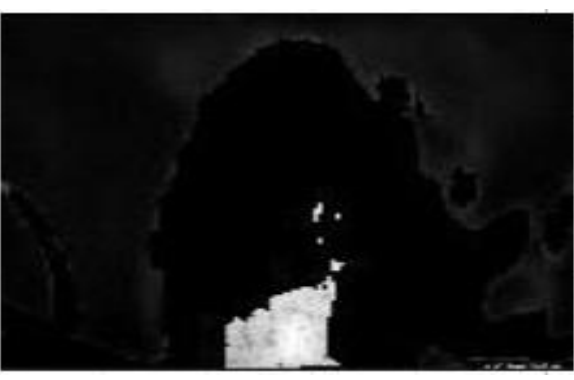

(c)

Fig. 9. Feature Extraction of Boll Weevil Attack on Cotton Buds (a) Input Image (b) Horizontal Gradient Extracted by Mathematical Morphology (c) Extracted Features using Proposed Method.

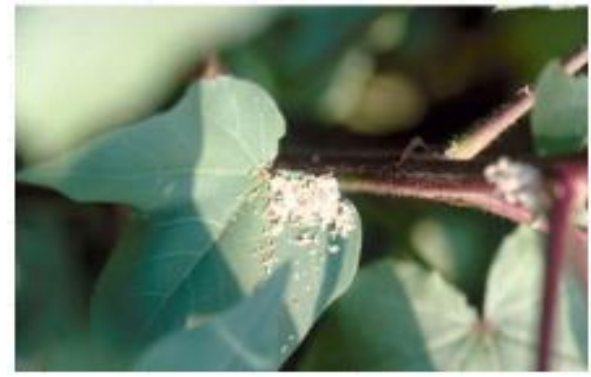

(a)

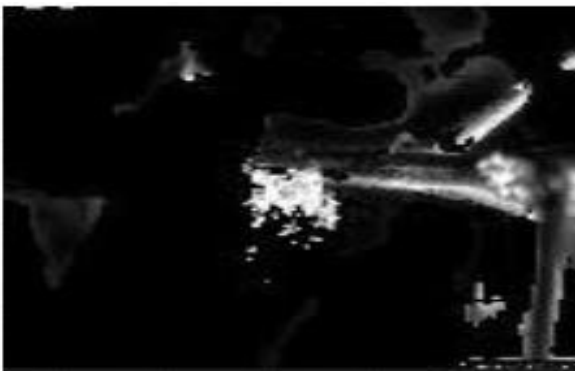

(b)

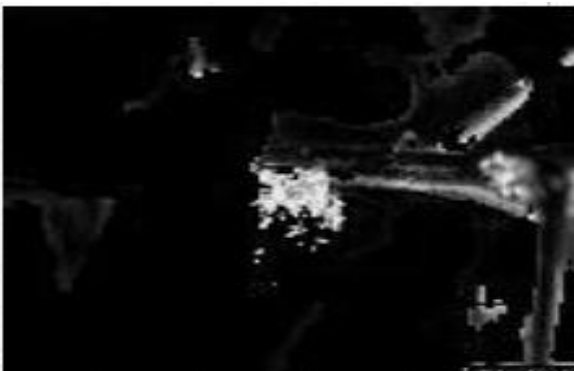

(c)

Fig. 10. Feature Extraction of European Corn Borer attack on Cotton Leaves (a) Input Image (b) Horizontal Gradient Extracted by Mathematical Morphology (c) Extracted Features using Proposed Method.

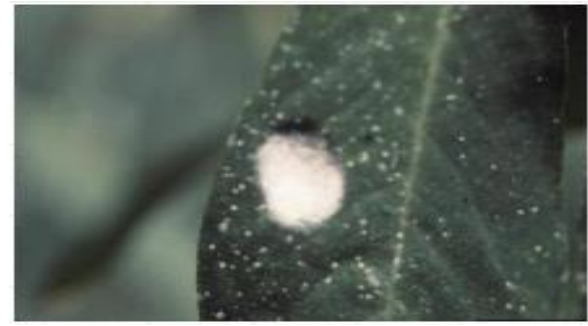

(a)

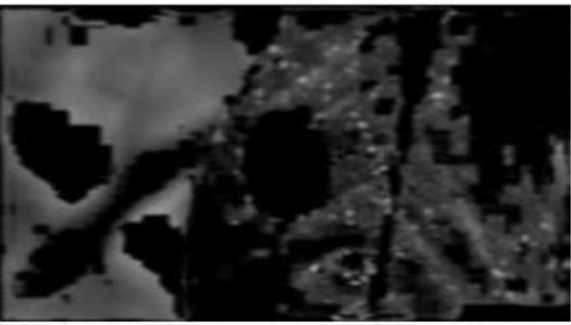

(b)

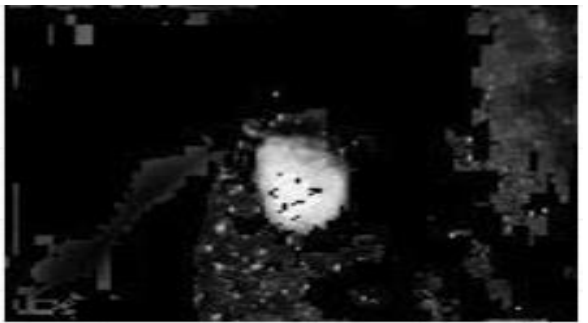

(c)

Fig. 11. Feature Extraction of Fall Army Worm attack on Peanut Leaves (a) Input Image (b) Horizontal Gradient Extracted by Mathematical Morphology (c) Extracted Features using Proposed Method.

TABLE. II. COMPARITIVE EVALUATION

\begin{tabular}{|l|l|l|}
\hline Method & Disease/Insect Attack & Accuracy \\
\hline Proposed Algorithm [FFTA+BPNN] & $\begin{array}{l}\text { Cercospora, Bacterial Blight, Boll Weevil, fall Army Worm, } \\
\text { European Corn Borer }\end{array}$ & $97.69 \%$ \\
\hline GLCM+SVM [9] & Bacterial Leaf Blight, Sheath Blight and Leaf Blast & $97.02 \%$ \\
\hline GLCM + BPNN [20] & $\begin{array}{l}\text { Frogeye Disease, } \\
\text { Downy Mildew and Bacterial Pustule }\end{array}$ & $93.03 \%$ \\
\hline GLCM+BPNN [1] & Bacterial Blight, Leaf Blast and Brown Spot & $89.60 \%$ \\
\hline Hu's Moment + BPNN [21] & Myrothecium, Bacterial Blight, and Alternaria & $85.52 \%$ \\
\hline
\end{tabular}

\section{CONCLUSION}

Plant disease detection is an important aspect in crop management for a stable crop production. In our proposed work, we have examined Enhanced Fusion Fractal Texture Analysis Technique for feature extraction. We have driven out a texture feature and selection method which combines SIFT and WSFTA extraction techniques and selects the best feature by performing PCA algorithm for further classification. From the testes that we have performed it is proven that the proposed method produces best result of $97.69 \%$ when extracted features are classified using BPNN. Compared to the existing traditional methods the proposed system has higher accuracy. Fusing more than two existing methods produces better result. We have used serial based fusion technique which extracts the similarity criteria among the individual algorithms. The tests were performed on more than 300 images which include multiple diseases and pest attack. The proposed method excelled in different factors including 
change of scale, Illumination, Gaussian blur effect etc. Including all the extracted features we have spotted the diseased regions on the leaves and the buds. It gives better result in low scaled images as well.

\section{REFERENCES}

[1] Huang KY (2007), "Application of artificial neural network for detecting Phalaenopsis seedling diseases using color and texture features," Computers and Electronics in Agriculture 57(1):3-11.

[2] Zhi-Kai Huang, Chun-Hou Zheng1, Ji-Xiang Du1, and Yuan-yuan Wan,Bark "Classification Based on Textural Features Using Artificial Neural Networks," ISNN 2006, LNCS 3972, pp. 355 - 360, 2006. (C) Springer-Verlag Berlin Heidelberg 2006.

[3] Dr. Shaik Asif Hussain, Raza Hasan, Dr. Shaik Javeed Hussain, "Classification and Detection of Plant Disease using Feature Extraction Methods," International Journal of Applied Engineering Research ISSN 0973-4562 Volume 13, Number 6 (2018) pp. 4219-4226 () Research India Publications. http://www.ripublication.com.

[4] Ashwini T Sapkal, Uday V Kulkarni, "Comparative study of Leaf Disease Diagnosis system usingTexture features and Deep Learning Features, International Journal of Applied Engineering Research, " ISSN 0973-4562 Volume 13, Number 19 (2018) pp. 14334-14340 @ Research India Publications, http://www.ripublication.com.

[5] Md. Junayed Hasan , Jia Uddin, "A Novel Modified SFTA Approach for Feature Extraction," iCEEiCT 2016, 978-1-5090-2906-8/16/\$31.00 (C)2016 IEEE.

[6] Chit Su Hlaing, Sai Maung Maung Zaw, "Tomato Plant Diseases Classification Using Statistical Texture Feature and Color Feature," 9781-5386-5892-5/18/\$31.00 @2018 IEEE, ICIS 2018, June 6-8, 2018, Singapore.

[7] K.Malathi, R.Nedunchelian, "Efficient Method To Detect And Classify Diabetic Retinopathy Using Retinal Fundus Images," International Journal of Pure and Applied Mathematics, Volume 116 No. 21 2017, 8997 ISSN: 1311-8080 (printed version); ISSN: 1314-3395 (on-line version).

[8] K Malathi1, R Nedunchelian, "A recursive support vector machine (RSVM) algorithm to detect and classify diabetic retinopathy in fundus retina images," Biomedical Research 2017, ISSN 0970-938X.

[9] Yao Q, Guan Z, Zhou Y, Tang J, Hu Y, Yang B (2009), “Application of support vector machine for detecting rice diseases using shape and color texture features," In: IEEE international conference on engineering computation ICEC, Hong Kong, pp 79-83.
[10] Pires RDL, Gonc alves DN, Orue ^ JPM, Kanashiro WES, Rodrigues JF, Machado BB, Gonc, alves WN (2016), "Local descriptors for soybean disease recognition", Comput Electron Agric 125:48-55.

[11] D.Saraswathi,G.Sharmila, E.Srinivasan, "An Automated Diagnosis system using Wavelet based SFTA Texture Features," ICICES2014, ISBN No.978-1-4799-3834-6,2014 IEEE.

[12] Attique Khan, Tallha Akram,Muhammad Sharif,Muhammad Younus Javed,Nazeer Muhammad, Mussarat Yasmin, "An implementation of optimized framework for action classification using multilayers neural network on selected fused features," Muhammad, Pattern Analysis and Applications, Springer-Verlag London Ltd., part of Springer Nature 2018.

[13] Chun-Lin, Liu, "A Tutorial of the Wavelet Transform," February 23, 2010, http://disp.ee.ntu.edu.tw/tutorial/WaveletTutorial.pdf.

[14] Plant Village Images, https://plantvillage.psu.edu/topics, Accessed 17 October 2019.

[15] Kapilya Gangadharan, G. Rosline Nesa Kumari, D. Dhanasekaran, "An Efficient Plant Disease Detection System Using Hybrid Watershed Segmentation with Extended K-Means Clustering Algorithm," International Journal of Advanced Science and Technology, Vol. 28, No. 11, (2019), pp.308-320).

[16] Kapilya Gangadharan, G. Rosline Nesa Kumari, D. Dhanasekaran, "Classification and Functional Analysis of Major Plant Disease using Various Classifiers in Leaf Images," International Journal of Innovative Technology and Exploring Engineering (IJITEE), ISSN: 2278-3075, Volume-9 Issue-2, December 2019.

[17] Kapilya Gangadharan, G. Rosline Nesa Kumari, D. Dhanasekaran, K.Malathi, "Plant Disease Diagnosis and Classification by Computer Vision using Statistical Texture Feature Extraction Technique and $\mathrm{K}$ Nearest Neighbor Classification," International Journal of Engineering and Advanced Technology (IJEAT), ISSN: 2249 - 8958, Volume-9 Issue-2, December, 2019.

[18] ForestryImages, https://www.forestryimages.org/series/series.cfm, Accessed23October 2019.

[19] Prasad S, Peddoju SK, Ghosh D (2016)," Multi-resolution mobile vision system for plant leaf disease diagnosis." Signal Image Video Process 10(2):379-388.

[20] Gharge S, Singh P (2016), "Image processing for soybean disease classification and severity estimation." In: Shetty N, Prasad N, Nalini N (eds) Emerging research in computing, information, communication and applications. Springer, New Delhi, pp 493-500.

[21] Rothe PR, Kshirsagar RV (2015)," Cotton leaf disease identification using pattern recognition techniques." In: IEEE international conference on pervasive computing (ICPC), January, pp 1-6. 